\title{
Influence of personal patterns of behavior on the effects of Tai Chi: a pilot study
}

\author{
Masahiro Toda $\cdot$ Rei Den $\cdot$ Masako Hasegawa-Ohira \\ Kanehisa Morimoto
}

Received: 3 March 2010/Accepted: 7 May 2010/Published online: 29 May 2010

(c) The Japanese Society for Hygiene 2010

\begin{abstract}
Objectives To investigate the influence of individual patterns of personality and behavior on the change in mood status after a brief period of Tai Chi exercise.

Methods The mood status in 22 healthy females was evaluated before and after a period of Tai Chi exercise using the Profile of Mood States (POMS) score. Patterns of personal behavior were also assessed by written questionnaire.

Results In the type A behavior pattern group, the score for total mood disturbance decreased significantly after a brief period (20 min) of Tai Chi exercise. No change was observed in the type B behavior pattern group.

Conclusions These findings suggest that a brief period of Tai Chi exercise is mentally beneficial, particularly to individuals with type A characteristics.
\end{abstract}

Keywords Tai Chi - POMS - Patterns of behavior . Mood status - Exercise

M. Toda $(\bowtie) \cdot$ R. Den $\cdot$ M. Hasegawa-Ohira $~ K$ K. Morimoto Department of Social and Environmental Medicine, Osaka University Graduate School of Medicine, 2-2 Yamada-oka, Suita, Osaka 565-0871, Japan e-mail: mt@envi.med.osaka-u.ac.jp

M. Toda

Department of Pharmacology, Osaka Dental University, Hirakata, Osaka, Japan

K. Morimoto

Twin Research Center, Osaka University Graduate

School of Medicine, Suita, Osaka, Japan

\section{Introduction}

Tai Chi has been receiving attention worldwide as an activity that promotes physical health and mental wellbeing. Tai Chi is a form of mobile meditation that combines moves from ancient Chinese martial arts with breath control and relaxation [1]. It is characterized by a series of slow body movements without a break or pause and can be enjoyed irrespective of age or gender. Many studies on the effects of Tai Chi have been conducted. In the long term, Tai Chi can favorably enhance cardiorespiratory function $[2,3]$ and may improve physical function, such as body balance $[4,5]$ or gait performance [6]. Furthermore, even in the form of brief exercise, it can improve self-reported mood and relieve stress [7, 8]. To the best of our knowledge, however, there have been no studies investigating individual variation in the effects of Tai Chi. In the pilot study reported here, we examined the influence of individual patterns of personality and behavior on how mood is affected by a brief period of Tai Chi exercise.

\section{Materials and methods}

This study was intended as a preliminary evaluation for a larger intervention study. Approval for the study was obtained from the Ethics Committee of the Osaka University, following which 22 healthy female volunteers (mean age 68.9 years) belonging to a local Tai Chi class were enrolled. Written informed consent was obtained from the subjects. The subjects performed classical Yangstyle Tai Chi, the most popular type, for 20 min, during which time they tried to imitate the instructor's motions and postures at the same speed and with the same technique. To evaluate their mood status quantitatively, before 
and after the exercise period, the subjects were asked to complete the 30-item Profile of Mood States (POMS) [9, 10], which is a self-rating questionnaire consisting of six mood dimensions: Tension-Anxiety (T-A), DepressionDejection (D), Anger-Hostility (A-H), Vigor (V), Fatigue (F), and Confusion (C). The total mood disturbance (TMD) score is calculated by subtracting the $\mathrm{V}$ score from the sum of scores for the other dimensions. As a control, the POMS questionnaire was completed at the same time on a day that the subjects did not have a Tai Chi class. Both experimental days were separated by just 1 week (starting at 1500 hours, Tuesday), and the order of the two measurements was randomized to cancel possible sequential effects.

Prior to the experiment, the participants were asked to complete a written questionnaire designed to assess patterns of behavior by means of the Tokai University Type A Pattern Scale [11, 12]. Based on the answers, the subjects were categorized as having either type A or type B behavior patterns. Health Practice Index (HPI) [13] scores were also measured to assess lifestyle.

Student's paired $t$ test was performed to compare mood status before and after Tai Chi exercise. Values were considered to be significantly different when $p<0.05$.

\section{Results}

Table 1 shows the basic characteristics of each group. Of the 22 subjects, 14 were classified as type A. There was no significant difference in age, height, body weight, or lifestyle between the two groups.

Table 2 shows the POMS scores for all subjects during the experimental period. For the type A behavior pattern group, after Tai Chi exercise, the scores for $\mathrm{D}(p<0.05)$, $\mathrm{A}-\mathrm{H}(p<0.001), \mathrm{F}(p<0.01)$, and $\mathrm{C}(p<0.05)$ as well as TMD $(p<0.05)$ decreased significantly. There was no such change in the type B group. During the control period, there was no significant change in the POMS scores for either type A or type B individuals.

\section{Discussion}

The D, A-H, F, and $\mathrm{C}$ scores of the type A behavioral group showed a statistically significant decrease after Tai Chi exercise. This was reflected in a statistically significant decrease in the TMD score. These findings suggest that, particularly for individuals with type A characteristics, Tai Chi improves mood. Tai Chi is a mind-body exercise with breath control, relaxation, and meditation [1] that may, therefore, soothe impatient individuals. In contrast, for type $\mathrm{B}$ individuals, who are characteristically calm by nature,
Table 1 Characteristics of the subjects

\begin{tabular}{lcl}
\hline Characteristics & $\begin{array}{l}\text { Type A behavior } \\
\text { pattern }(n=14)\end{array}$ & $\begin{array}{l}\text { Type B behavior } \\
\text { pattern }(n=8)\end{array}$ \\
\hline Age (years) & $69.9 \pm 7.2$ & $67.3 \pm 8.6$ \\
Height (cm) & $151.7 \pm 5.3$ & $152.4 \pm 5.3$ \\
Body weight (kg) & $51.4 \pm 10.9$ & $49.8 \pm 4.7$ \\
Health Practice Index (HPI) & $6.5 \pm 1.1$ & $6.6 \pm 1.1$ \\
Percentage of respondents with good lifestyle & \\
$\begin{array}{l}\text { Smoking habits } \\
\text { Drinking habits }\end{array}$ & 100 & 100 \\
$\begin{array}{l}\text { Daily consumption of } \\
\text { breakfast }\end{array}$ & 92.9 & 100 \\
Appropriate daily duration & 92.9 & 100 \\
$\quad$ of sleep & 50.0 & 50.0 \\
Appropriate daily duration & 92.9 & 75.0 \\
$\quad$ of work & & 100 \\
$\begin{array}{l}\text { Regular physical activity } \\
\text { Appropriate levels } \\
\quad \text { of subjective stress }\end{array}$ & 92.9 & 87.5 \\
Nutritionally balanced diet & 42.9 & 50.0 \\
\hline
\end{tabular}

Where applicable, values are given as the mean \pm standard deviation (SD)

the mood effects of Tai Chi are less obvious. A previous study found significantly higher POMS scores for individuals with type A characteristics [14]. In our study, before the Tai Chi exercise (baseline), the POMS scores in question (D, A-H, F, and C) tended to be somewhat higher in the behavioral type A group than in the type B group, but this difference was not statistically significant. However, the lack of effect in the type B group may have resulted from a small sample size $(n=8)$. Larger populations are needed for future studies. In addition, since the intensity of Tai Chi exercise is comparable to that of brisk walking [7], these results could also be interpreted as resulting from mere physical activity. However, in a number of previous studies, POMS scores were found not to be affected by brisk walking [15-17].

We failed to find any significant change in the T-A score in the type A group following a brief period of Tai Chi exercise. This may have been due to how the Tai Chi exercise was conducted: subjects doing their best to imitate the instructor's motions and postures at the same speed and fine detail. It is possible that the tension of type A subjects persisted throughout the 20-min period of exercise. In addition, we found no significant change in $\mathrm{V}$ scores. In contrast, Jin studied 24 Tai Chi practitioners and reported an enhancement of vigor after $60 \mathrm{~min}$ of Tai Chi exercise [7]. This discrepancy in results may possibly be due to the shorter exercise period in our study. Further investigation is required to clarify this point. 
Table 2 Changes in mood scores elicited before and after Tai Chi exercise

\begin{tabular}{|c|c|c|c|c|c|c|c|c|}
\hline \multirow[t]{3}{*}{ POMS } & \multicolumn{4}{|c|}{ Tai Chi exercise } & \multicolumn{4}{|l|}{ Control } \\
\hline & \multicolumn{2}{|c|}{$\begin{array}{l}\text { Type A behavior } \\
\text { pattern }(n=14)\end{array}$} & \multicolumn{2}{|c|}{$\begin{array}{l}\text { Type B behavior } \\
\text { pattern }(n=8)\end{array}$} & \multicolumn{2}{|c|}{$\begin{array}{l}\text { Type A behavior } \\
\text { pattern }(n=14)\end{array}$} & \multicolumn{2}{|c|}{$\begin{array}{l}\text { Type B behavior } \\
\text { pattern }(n=8)\end{array}$} \\
\hline & Before & After & Before & After & Before & After & Before & After \\
\hline $\mathrm{T}-\mathrm{A}$ & $44.4 \pm 7.1$ & $40.9 \pm 4.2$ & $41.5 \pm 4.2$ & $43.0 \pm 6.5$ & $41.9 \pm 5.6$ & $42.1 \pm 4.1$ & $42.0 \pm 7.1$ & $41.1 \pm 4.0$ \\
\hline D & $45.3 \pm 5.5$ & $41.6 \pm 2.1 *$ & $43.4 \pm 3.7$ & $46.0 \pm 6.0$ & $43.3 \pm 4.0$ & $44.6 \pm 2.3$ & $43.5 \pm 6.0$ & $43.6 \pm 2.6$ \\
\hline A-H & $44.8 \pm 5.8$ & $38.8 \pm 1.6^{* * *}$ & $42.0 \pm 4.5$ & $42.1 \pm 4.1$ & $42.1 \pm 4.0$ & $41.7 \pm 1.3$ & $43.5 \pm 5.7$ & $42.0 \pm 2.8$ \\
\hline V & $48.6 \pm 12.8$ & $48.3 \pm 12.4$ & $49.4 \pm 11.0$ & $51.9 \pm 10.8$ & $55.1 \pm 11.2$ & $53.4 \pm 8.8$ & $41.9 \pm 7.1$ & $43.8 \pm 9.1$ \\
\hline $\mathrm{F}$ & $43.7 \pm 5.0$ & $39.1 \pm 2.7 * *$ & $43.4 \pm 3.2$ & $42.0 \pm 5.2$ & $42.1 \pm 3.6$ & $42.1 \pm 1.9$ & $43.5 \pm 5.0$ & $43.3 \pm 3.2$ \\
\hline $\mathrm{C}$ & $46.5 \pm 7.1$ & $42.4 \pm 5.6^{*}$ & $43.4 \pm 3.9$ & $47.5 \pm 7.5$ & $43.9 \pm 4.2$ & $42.6 \pm 3.9$ & $43.4 \pm 6.2$ & $41.8 \pm 4.3$ \\
\hline TMD & $176.1 \pm 30.8$ & $154.5 \pm 9.1^{*}$ & $164.3 \pm 18.4$ & $168.8 \pm 34.3$ & $158.3 \pm 20.5$ & $159.6 \pm 10.7$ & $174.0 \pm 26.8$ & $168.0 \pm 9.8$ \\
\hline
\end{tabular}

Values are expressed as the mean $\pm \mathrm{SD}$

POMS Profile of Mood States, $T-A$ tension-anxiety, $D$ depression-dejection, $A-H$ anger-hostility, $V$ vigor, $F$ fatigue, $C$ confusion, $T M D$ total mood disturbance

$* p<0.05, * * p<0.01, * * * p<0.001$ (Student's paired $t$ test): in terms of being significantly different from "before Tai Chi exercise"

In this pilot study, we found that a brief period ( $20 \mathrm{~min})$ of Tai Chi exercise was mentally beneficial, particularly to individuals who fall into the behavioral type A category, which is associated with higher coronary risk [18]. A number of previous studies have reported that long-term Tai Chi practice can favorably enhance cardiorespiratory function [2,3]. Taken together, these findings suggest that Tai Chi may be helpful in preventing coronary heart disease. Other previous studies have found that short- or longterm Tai Chi exercise can decrease the levels of a physiological stress marker, cortisol [7, 8, 19]. To further elucidate individual variation in the mental effects of Tai Chi and its mechanism, we are planning further investigations using a more multifaceted approach, including objective markers.

\section{References}

1. Delza S. T'ai Chi Ch'uan: body and mind in harmony (integration of meaning and method). New York: SUNY Press; 1985.

2. Lai JS, Lan C, Wong MK, Teng SH. Two-year trends in cardiorespiratory function among older Tai Chi Chuan practitioners and sedentary subjects. J Am Geriatr Soc. 1995;43:1222-7.

3. Lan C, Chen SY, Lai JS, Wong MK. The effect of Tai Chi on cardiorespiratory function in patients with coronary artery bypass surgery. Med Sci Sports Exerc. 1999;31:634-8.

4. Maciaszek J, Osiński W, Szeklicki R, Stemplewski R. Effect of Tai Chi on body balance: randomized controlled trial in men with osteopenia or osteoporosis. Am J Chin Med. 2007;35:1-9.

5. Tsang WW, Hui-Chan CW. Effect of 4- and 8-wk intensive Tai Chi Training on balance control in the elderly. Med Sci Sports Exerc. 2004;36:648-57.

6. Shen CL, James CR, Chyu MC, Bixby WR, Brismée JM, Zumwalt MA, Poklikuha G. Effects of Tai Chi on gait kinematics, physical function, and pain in elderly with knee osteoarthritis: a pilot study. Am J Chin Med. 2008;36:219-32.

7. Jin P. Changes in heart rate, noradrenaline, cortisol and mood during Tai Chi. J Psychosom Res. 1989;33:197-206.

8. Jin P. Efficacy of Tai Chi, brisk walking, meditation, and reading in reducing mental and emotional stress. J Psychosom Res. 1992;36:361-70.

9. McNair D, Lorr M, DroppLemm L. Manual for the Profile of Mood States (POMS). San Diego: Educational and Industrial Testing Service; 1971.

10. Yokoyama K, Araki S. Manual of Japanese version of POMS. Tokyo: Kaneko Syobo; 1994 (in Japanese).

11. Hosaka T, Tagawa R. The Japanese characteristic of Type A behavior pattern. Tokai J Exp Clin Med. 1987;12:287-303.

12. Hosaka T, Tagawa R. The coronary-prone behavior pattern among Japanese: its comparison with Type A behavior pattern. Jpn J Psychosom Med. 1989;29:527-36.

13. Morimoto K. Lifestyle and health. Jpn J Hyg. 2000;54:572-91.

14. Fukunishi I, Hattori M. Mood states and Type A behavior in Japanese male patients with myocardial infarction. Psychother Psychosom. 1997;66:314-8.

15. Cramer SR, Nieman DC, Lee JW. The effects of moderate exercise training on psychological well-being and mood state in women. J Psychosom Res. 1991;35:437-49.

16. Nieman DC, Custer WF, Butterworth DE, Utter AC, Henson DA. Psychological response to exercise training and/or energy restriction in obese women. J Psychosom Res. 2000;48:23-9.

17. Stanton JM, Arroll B. The effect of moderate exercise on mood in mildly hypertensive volunteers: a randomized controlled trial. J Psychosom Res. 1996;40:637-42.

18. Tagawa R, Hosaka T. Study of the correlation between the Type A behavior pattern in patients with coronary heart disease and the extent of coronary atherosclerosis. Tokai J Exp Clin Med. 1990;15:45-50.

19. Esch T, Duckstein J, Welke J, Braun V. Mind/body techniques for physiological and psychological stress reduction: stress management via Tai Chi training: a pilot study. Med Sci Monit. 2007;13:CR488-97. 